\title{
La aparición de las redes sociales como contenido informativo en los medios tradicionales: análisis comparativo en dos diarios españoles de referencia (2006-2010)
}

\author{
Natalia MELÉndez MaLAVÉ \\ Universidad de Málaga \\ natalia@uma.es \\ Antonio CUARTERo NARANJO \\ Universidad de Málaga \\ ant.cuartero@gmail.com
}

Recibido: 05/06/2012

Aceptado: 17/01/2013

\begin{abstract}
Resumen
Las redes sociales son un fenómeno de aparición reciente pero de gran impacto, y su reflejo como contenido informativo es una clave para entender cómo se está percibiendo esta nueva realidad y, sobre todo, cómo la han acogido los medios tradicionales. Como respuesta se presenta un estudio comparativo de dos diarios españoles de referencia-El País y El Mundo- en los que se investiga el número de noticias aparecidas sobre las redes más populares (Facebook, Twitter y Tuenti), en su primer lustro de existencia, analizándolas según su enfoque, su procedencia, sus temáticas y su ubicación. Además se contrasta la postura que adoptan estos medios ante las distintas redes sociales, y el perfil y personalidad, muy marcado, que se traslada de cada una de ellas.
\end{abstract}

Palabras clave: redes sociales; prensa de referencia; Internet; web 2.0; representación mediática.

Appearance of Social Networks in Traditional Media as Informative Content: Comparative Analysis from two Spanish Quality Newspapers (2006-2010)

\begin{abstract}
Social networks are a phenomenon of recent appearance but that had have a great impact and its reflection as information content is a key to understand how this new reality is perceived and, most of all, how traditional media has received it. As a reply, we present a comparative study of two Spanish broadsheets -El País and El Mundo- in which we analyze the number of news published about the most popular social networks (Facebook, Twitter and Tuenti) in the first five years of their existence, analysing about its approach, its origin, its themes and its location. Furthermore we made a comparison about the position that these journals adopt in view of the different social network, and the profile and personality, strongly pronounced, that it is communicated of each one.
\end{abstract}

Keywords: Social networks; quality press; Internet; Web 2.0; Media representation.

\section{Referencia normalizada}

MELÉNDEZ MALAVÉ, Natalia Y CUARTERO NARANO, Antonio (2013): “La aparición de las redes sociales como contenido informativo en los medios tradicionales: análisis comparativo en dos diarios españoles de referencia (2006-2010)". Estudios sobre el mensaje periodístico. Vol. 19, Núm. 1, págs.: 177-189. Madrid, Servicio de Publicaciones de la Universidad Complutense.

Sumario: 1. Introducción. 2. Metodología. 3. Resultados; 3.1. Enfoque; 3.2. Temáticas; 3.3. Secciones. 4. Conclusiones. 5. Referencias bibliográficas. 


\section{Introducción}

A tenor de la dimensión que ha alcanzado el fenómeno de las redes sociales por internet podría decirse que éstas parecen destinadas a heredar el cometido de un "quinto poder" en la línea proyectada por Ignacio Ramonet cuando hace algunos años planteaba ya la necesidad de "oponer una fuerza cívica ciudadana a la nueva coalición dominante. Un quinto poder cuya función sería denunciar el superpoder de los medios de comunicación, de los grandes grupos mediáticos, cómplices y difusores de la globalización liberal". (Ramonet, 2004: web).

El impacto que ha supuesto su aparición y desarrollo posterior es indiscutible, ya que los llamados social media han transformado nuestra forma de relacionarnos y de intercambiar información ${ }^{1}$. Además de lo singular de la rápida y masiva adaptación de los internautas: los datos de ComScore afirman que a finales de 2010 existían 945 millones de usuarios de redes sociales en el mundo y sólo en España, en mayo de 2011, 22 millones de visitantes únicos invirtieron más del $23 \%$ del total de su tiempo navegando por internet en el uso de redes sociales ${ }^{3}$. La misma fuente indica que en España plataformas como Facebook o Tuenti han alcanzado una gran aceptación al contar con 15 y con 7,7 millones de usuarios, respectivamente. Además, según los datos de Alexa, Facebook ocupa el segundo lugar en la clasificación de los sitios más visitados tanto en España como a nivel mundial y Twitter ocupa la novena posición en los dos $\operatorname{casos}^{4}$.

La investigación sobre estas plataformas de contacto, que constituyen una de las señas de identidad de la web 2.0, se ha centrado en buena medida en la descripción de sus características, en su aplicación para el estudio del funcionamiento de las redes, en sus posibilidades de negocio o de influencia política, y, por supuesto, en cuantificar sus usuarios o detectar sus perfiles. Pero, centrándonos exclusivamente en la perspectiva de la comunicación social, no cabe duda de que el fenómeno conlleva unas consecuencias para la forma de concebir el periodismo, la actualidad o los medios, sobre la que nos proponemos indagar con el presente trabajo. En especial, nos interesa conocer cómo han acogido los medios convencionales -la prensa en este caso- la llegada de esta nueva vía de comunicación que es a la vez un apoyo y una competencia.

En efecto, como afirma Bacallao Pino (2010: web) estas tecnologías de la comunicación suponen una dimensión de la realidad directamente vinculada a los medios, pero, al mismo tiempo resultan un espacio en el cual emergen y se van gestando otras dinámicas comunicativas globales, que conviven, en ese mundo llamado internet, con los media. A su vez, según el mismo autor, "ello supone una relación tensa de proxi-

1 Para un análisis detallado de las múltiples repercusiones sociales del fenómeno, véase: Cachia. Romina, (2008) "Los sitios de creación de redes: aspectos sociales", en Telos: Cuadernos de comunicación e innovación, $\mathrm{N}^{\mathrm{o}}$. 76, pp. 69-84.

2 http://www.comscore.com/Press_Events/Press_Releases/2010/8/Facebook_Captures_Top Spot_among_Social_Networking_Sites_in_India [Fecha de consulta: 24 de octubre de 2011]

3 Según las mediciones ofrecidas en: http://www.comscore.com/esl/Press_Events/Press_Releases/2011/7/comScore_Releases_Overview_of_European_Internet_Usage_for_May_2011 .[Fecha de consulta: 24 de octubre de 2011]

4 Datos extraídos de http://www.alexa.com/topsites y http://www.alexa.com/topsites/countries/ES. [Fecha de consulta: 31 de octubre de 2011] 
midad y diferencia, de cercanía y distanciamiento" porque a pesar de presentar cualidades opuestas a los medios de comunicación, estos últimos se ven obligados a adaptarse a ellas.

Ejemplo de esto es el hecho de que Twitter está suponiendo un instrumento para la circulación rápida de noticias, como se ha comprobado en casos recientes como el movimiento del 15-M o las revueltas y protestas en el mundo árabe. Pero al mismo tiempo existe el riesgo para los medios convencionales de que las redes sociales se les adelanten al proporcionar informaciones por parte de los propios usuarios, como ya se ha dado en algún caso ${ }^{5}$. Con todo, como manifiesta Campos Freire (2008: web), "las redes se aprovechan directa o indirectamente del valor informativo generado por los medios mientras que el uso que hacen éstos de la llamada Web 2.0 o Web social es mucho más conservador y proteccionista con respecto a su propio modelo".

Esta dualidad, según la cual medios convencionales y redes se necesitan a la vez que se oponen, no ha hecho más que dar sus primeros pasos, como recoge Juan Ramón Sánchez (2008: 73), según el cual "los impactos del éxito de la Web 2.0 y en particular de las redes sociales sobre los medios tradicionales de comunicación están lejos de haberse agotado". El mismo autor acto seguido hace hincapié en los efectos del periodismo ciudadano propugnado por Dan Gillmor, en el que los usuarios asumen roles que hasta ahora sólo se ejercían desde la práctica profesional del periodismo. En este sentido, siguiendo a Campos Freire (2008: web), "el modelo de actividad -y por supuesto de organización y negocio- de los medios tradicionales es muy diferente. El sistema informativo de los medios sigue el dictado del modelo tradicional de pilotaje: selección, evaluación y control de los contenidos. Este no es el modelo que casa, precisamente, con la filosofía colaborativa, abierta, horizontal y participativa de la red social'.

Al mismo, tiempo, los medios continúan ejerciendo su papel como agente configurador de mentalidades y de representación de la realidad. Como nos recuerda Miquel Rodrigo (1989: 31) "a los periodistas se les atribuye la competencia de recoger los acontecimientos y temas importantes y atribuirles un sentido. Este contrato se basa en unas actitudes epistémicas colectivas que se han ido forjando por la implantación del uso social de los medios de comunicación como transmisores de la realidad social de importancia pública". Por este motivo, la pretensión inicial de esta investigación, la constituye, pues, comprobar qué informaciones recoge la prensa acerca de las redes sociales y, por consiguiente, qué visión de ellas trasladan estas noticias. Se persigue obtener de este modo una perspectiva del fenómeno mediante su reflejo en el periodismo impreso y, para ello, este propósito de carácter general se desgrana en una serie de objetivos más específicos:

- Cuantificar el número de noticias que se publican sobre las redes sociales desde los primeros momentos de su aparición como objeto de interés informativo y si la responsabilidad de la producción de dichos contenidos es interna o externa al medio.

5 La red social Twitter abordó casi instantáneamente el amerizaje de un avión accidentado en el río Hudson y fue la primera en publicar una foto del suceso, según se recogió posteriormente en algunos medios: http://www.telegraph.co.uk/technology/twitter/4269765/NewYork-plane-crash-Twitter-breaks-the-news-again.html. 
- Conocer la postura que adopta cada medio seleccionado ante la irrupción de estas nuevas vías comunicativas.

- Identificar la ubicación de las informaciones relativas a las redes sociales dentro de la tematización de la realidad que efectúa el periódico a través de sus secciones.

- Conocer las diferentes categorías temáticas a las que se ajustan las noticias sobre las redes sociales, toda vez que los contenidos relacionados con ellas comprenden un espectro amplio que va desde las novedades de los social media en tanto empresas o las aplicaciones tecnológicas que van incorporando, hasta el uso que hacen de ellas las audiencias, entre otras cuestiones.

- Describir el perfil y características que los periódicos seleccionados proyectan de las plataformas de contacto más populares.

\section{Metodología}

Dado que nuestro objetivo prioritario consistía en realizar una radiografía de la visión que ofrece la prensa sobre las redes sociales, nuestro primer problema lo constituyó la selección de un corpus de noticias a analizar. Se tomó la decisión de trabajar con las ediciones electrónicas de los dos periódicos generalistas nacionales con mayor difusión, El País y El Mundo, pertenecientes a grupos de comunicación distintos (PRISA, Unidad Editorial) y que representan líneas diferentes que permiten la elaboración de un análisis comparativo ${ }^{6}$. Dentro del soporte digital se trabajó con los mismos contenidos de la publicación en papel, pero la búsqueda de casos que se correspondiesen con nuestro tema de interés resultaba más operativa a través de los servicios de hemerotecas digitales de ambos diarios.

Para obtener, pues, los textos que hicieran referencia al descriptor "redes sociales", utilizar dicha expresión como término de búsqueda resultaba problemático dada su ambivalencia. Así pues, se determinó buscar las noticias que contuviesen los nombres de las tres plataformas más populares en España ${ }^{7}$ : Facebook, Twitter y Tuenti ${ }^{8}$. Evidentemente esta última no tiene la fuerza y presencia de las otras dos redes internacionales, mucho más poderosas, pero era lógico prestarle atención a una empresa española, que además goza de un seguimiento muy continuo por parte de los medios

${ }^{6}$ Es indiscutible que se trata de dos cabeceras de referencia, pero, en todo caso, incluimos el dato objetivo del promedio de difusión de ambos periódicos según la OJD para el periodo entre enero y diciembre de 2010: 307.080 diarios en el caso de El País y 284.901 para El Mundo.

7 Según AIMC en su estudio anual de "Navegantes en la red" 2011, Facebook es la red más utilizada, en concreto, por el $90 \%$ de los usuarios. Le siguen a esta Tuenti con un $26,2 \%$ y Twitter con un $21,9 \%$.

8 En un primer momento se barajó incluir también MySpace, pero la búsqueda de noticias arrojó unos resultados escuetos y en declive, como la propia pujanza de la plataforma, a pesar de ser una de las más veteranas -su lanzamiento data de 2003- pero en los últimos años está experimentando, junto a otras redes como Hi5 o Fotolog una dinámica negativa, con las mayores tasas de abandono de redes, por encima del $20 \%$ y por tercer año consecutivo sufriendo una drástica pérdida de usuarios, según la tercera oleada del Observatorio de Redes Sociales de la consultoría The Cocktail Analysis (febrero 2011). http://www.tcanalysis.com /uploads/2011/02/Observatorio-RedesSociales2011.pdf 
de comunicación y que está situada en la decimoséptima posición entre las webs más visitadas de España según Alexa.

En cuanto al periodo de análisis también se nos planteaban distintos problemas. La delimitación de la fecha inicial estaba marcada por el año de lanzamiento de cada red social. La primera de las tres redes sociales en nacer es Facebook, que empieza a funcionar en Estados Unidos en 2004. Por su parte, Tuenti y Twitter aparecen en 2006. Así pues, la creación de estas dos últimas en dicho año fijó nuestro punto de partida, dado que en los dos primeros años de vida de Facebook no hay ninguna referencia en la prensa revisada. Además, tal y como indica Campos Freire (2008): "la llamada Web 2.0 , que acuña Tim O'Really en 2003, no se populariza ni caracteriza como "red social" ni como nueva filosofía colaborativa de hacer las cosas, hasta 2005/06. Es un fenómeno de emergencia muy reciente".

En consonancia con esto, no es hasta 2006 cuando comenzamos a obtener resultados de aparición de noticias en los medios elegidos en los que estas redes ocupan un papel central protagonizando la información. Así pues, se analizaron las noticias aparecidas entre enero de 2006 y diciembre de 2010 para cerrar un ciclo completo de un lustro.

Con estos primeros cinco años de información sobre redes sociales en El País y El Mundo manejábamos un enorme volumen de noticias relacionadas con las redes sociales, en total 6.486 piezas, de las que finalmente se analizaron 1.157. Esta reducción del corpus, que lo hacía además más manejable, es el resultado de aplicar dos criterios que debían cumplir las informaciones: en primer lugar, que se tratase de artículos que no formasen parte de los contenidos complementarios ofrecidos por la versión digital de cada periódico (como por ejemplo, entradas de blogs asociados a cada cabecera), es decir, que se trabajase con material tal y como el que aparece en el soporte papel. El segundo filtro consistía en que se tratase de informaciones cuyo tema principal o cuyo protagonismo perteneciera a alguna de las tres redes sociales seleccionadas, esto es, que no se las nombrara tangencialmente. Todas aquellas noticias en las que lo que se hacía era una mera referencia a las redes sociales o aparecían como tema secundario eran descartadas.

Una vez elegidas las redes sociales a analizar, el marco temporal y el corpus de piezas periodísticas en los correspondientes diarios, se procedió a la confección de una ficha de análisis para la extracción de datos. Se configuraron tres grandes bloques, un primer bloque identificativo de la noticia, en el que se incluyó el titular, el texto completo, la fecha, la autoría y la sección donde se ubica dentro del periódico. De esta forma el conjunto de fichas de análisis a la vez se utilizó como base de datos para poder hacer los posteriores cruces de datos y extraer resultados. El segundo bloque se centró en el enfoque de la noticia. Este es uno de los aspectos más importantes del análisis y cumple con uno de nuestros objetivos principales, conocer qué perspectiva aportan estos dos periódicos sobre las distintas redes sociales, clasificando este punto de vista como positivo, negativo o neutral. El tercer y último bloque tenía por objeto elaborar una categorización temática de las noticias a analizar. Se decidió, tras hacer un inicial pre-test, que los temas más recurrentes y que se ajustaban a nuestro análisis y objetivos se podían encuadrar en siete categorías, bajo los siguientes epígrafes: 
Empresarial, Tecnología y aplicaciones, Usuarios, Privacidad, Delincuencia, Activismo, Imagen pública, corporativa e institucional y Libertad de expresión. El conjunto de los contenidos que engloban merece, a nuestro juicio, una somera justificación:

El apartado denominado Empresarial se dedicó a las noticias que tienen que ver con las redes sociales en tanto que corporaciones: sus movimientos, sus estrategias, sus operaciones. También hemos incluido dentro de este apartado todo lo relacionado con la figura de sus dirigentes, así, por ejemplo, las muchas referencias al creador de Facebook, Mark Zuckerberg, se engloban dentro de este apartado. También se han incluido todas las noticias que tienen que ver con la irrupción de las redes sociales como fenómeno en la sociedad.

El ítem Tecnología y aplicaciones incluye todas las informaciones referidas a nuevos desarrollos y servicios diseñados para mejorar las redes y que periódicamente son publicitados por las propias empresas y objeto de atención de los medios.

En Usuarios hemos incluido todo tipo de noticias que tenían que ver tanto con personajes anónimos cuyo perfil cobra eco en un momento determinado, como con personajes famosos o importantes que hacen uso de las redes sociales. También cuando nos referimos a usuarios incluimos los empleos espurios que tienen que ver con filtraciones, por ejemplo, casos de reuniones que son a puerta cerrada y cuyo contenido se hace público a través de las redes. Asimismo, ciertas informaciones sobre aspectos cuantitativos relacionados con las audiencias conseguidas por las redes sociales forman parte también de las noticias recogidas bajo este epígrafe.

La cuestión de la Privacidad merecía conformar un epígrafe propio, dado su cariz controvertido. Las noticias que hemos incluido aquí se refieren a todas aquellas en las que de alguna forma se vulnera la intimidad de los usuarios a través de las redes sociales. Y también a todas aquellas noticias alusivas a los derechos de autor o derechos de los usuarios. En esta sección también hemos incluido todas las referencias al llamado "ciberacoso".

En cuanto a otro de los temas más polémicos cuando se habla de redes sociales, el ítem Delincuencia, tenemos que especificar que las noticias que hemos englobado dentro de este tema son exclusivamente informaciones relacionadas con usuarios que utilizan las redes sociales para cometer algún tipo de delito. Utilizan las vías que le ofrecen las redes para cometer algún acto ilegal. Esto se traduce en que acontecimientos relacionados con la comisión de delitos fuera de la red y publicitados a través de ella no eran considerados dentro de esta categoría.

El tema Activismo comprende todas las noticias referidas a iniciativas individuales o grupales de ciudadanos anónimos que a través de las redes intentan conseguir un objetivo a través de peticiones de ayuda, convocatorias de manifestaciones, creación de grupos para una causa común, etc. explotando así la capacidad movilizadora de las redes sociales.

En Imagen pública, corporativa e institucional hemos incluido todas aquellas noticias en las que alguna empresa, partido o entidad emplea las redes sociales como vehículo para vender o publicitar su imagen. Algo cada vez más frecuente dentro de estos entornos y que muchas marcas y organizaciones utilizan progresivamente. 
En Libertad de expresión se han incluido todas las noticias que tienen que ver con limitaciones a este derecho. Este tipo de informaciones se dan sobre todo en el plano internacional y en países donde la libertad de expresión tiene ciertas limitaciones o restricciones.

\section{Resultados}

Se han analizado un total de 1.157 noticias, de las cuales 650 se publicaron en El País y 507 en El Mundo. Aunque es un número más o menos equilibrado de noticias en ambos periódicos, podemos explicar la sutil diferencia en el hecho de que la atención por parte de El País a las redes sociales es más temprana que en El Mundo. En este periódico se obtienen resultados en las tres redes a partir del año 2009 mientras que en El País desde el año 2007 y 2008 ya se encuentran noticias de Facebook y Tuenti.

Como excepción tenemos que destacar que en el caso de Twitter obtenemos resultados mucho más tarde, pues su nacimiento en Estados Unidos y su popularización en España -tras el lanzamiento de la versión en español en noviembre de 2009- son más tardíos que en el caso de las otras dos redes sociales.

A pesar de ser la más joven de las tres redes sociales analizadas, Twitter, ha experimentado una gran progresión con respecto a la atención mediática que suscita. En el resultado final de las noticias analizadas queda por encima de Tuenti y muy cerca de Facebook.

En definitiva, Facebook es la red social con más presencia en ambos medios, duplica el número de noticias de la red española y supera en cerca de cien a la de $m i$ croblogging. Entre otros motivos, la explosión de la popularidad mediática de Facebook se debe tanto al rodaje y estreno de la película sobre su gestación, La red social, como a la popularidad de su creador, Mark Zukerberg, cuya controvertida figura es, a diferencia de la de otros gurús de la Web 2.0, de sobra conocida.

En cuanto a la autoría de las informaciones hay que destacar la diversa cantidad de firmas dentro de estas noticias, lo que provoca un enorme volumen de datos. En rasgos generales, podemos decir que un tercio de las noticias analizadas corresponden a la reelaboración de un teletipo, ya que vienen firmadas por la propia redacción de los periódicos o con una firma doble compartiendo la autoría con algunas de las agencias informativas más importantes. Otro tercio de las noticias provienen exclusivamente de las principales agencias, EFE, Reuters, AFP, etc. El Mundo muestra un uso más alto de las agencias de noticias en este tipo de informaciones que El País.

Ello se explica en parte porque, aunque, como vemos, ambos diarios recurren poco a la elaboración propia en las noticias analizadas, en el caso de El País sí podemos destacar a una periodista, Rosa Jiménez Cano, como la encargada en más ocasiones de tratar este tipo de temas, con un $9 \%$ del total de noticias de El País. De estos primeros datos puede interpretarse que al ser todavía un fenómeno joven, y al no tener aún los medios muy claro dónde ubicar estas noticias, es frecuente que no haya redactores especializados en estos temas. Además, el carácter transnacional de las redes sociales hace que muchas informaciones relativas a ellas procedan de cualquier punto del mundo y por lo tanto sean de procedencia externa, al igual que todas aquellas piezas informativas vinculadas a la estrategia corporativa de las dos empresas radicadas en Estados Unidos, Facebook y Twitter. 
En cualquier caso, estas circunstancias provocan que buena parte de las noticias sean breves, poco profundas y sin contextualizar, de tal manera que las informaciones carecen de seguimiento, quedando muchas de ellas aisladas, casi como curiosidades o anécdotas de consumo rápido -incluso muchas de ellas alcanzan el ranking de las noticias más leídas- pero cuentan historias de las que los lectores no volverán a saber.

\subsection{Enfoque}

En el cómputo total de la imagen de las tres redes sociales analizadas en los dos periódicos, resulta significativo que casi la mitad, el $46 \%$, de las noticias tengan un enfoque negativo mientras que solamente el $19 \%$ ofrecen una visión positiva de las redes sociales. Incluso las noticias que se han considerado como neutrales superan a las de carácter positivo, con un 35\% de noticias en las que se valoran a la vez tanto aspectos favorables como desfavorables de las redes sociales, aportando una perspectiva más equilibrada.

Descendiendo un escalón más en la interpretación de estos datos, en los dos periódicos estudiados hay ciertas diferencias. En El País se dan más casos de noticias positivas, con un 24\%, mientras que en El Mundo solo hay un 13\% y, más aún, en este último periódico más de la mitad de las noticias, el $56 \%$, son de carácter negativo, frente a un $37 \%$ en el caso de El País.

La red peor valorada en ambos diarios es Tuenti, con un enfoque negativo en el $51 \%$ de los casos en El País y el $46 \%$ en El Mundo. Las razones de estos altos índices de una orientación crítica hacia la red española en estos periódicos se deben a varias causas. Por un lado, encontramos el hecho de que, especialmente en El Mundo, las noticias sobre Tuenti no aparezcan mayoritariamente en la sección de Tecnología, que es -como veremos más abajo- la sección natural de estas noticias en el periódico. Así, resulta significativo que las informaciones que mencionan Tuenti aparezcan relacionadas con noticias de sucesos. Tuenti desde sus inicios hasta hoy ha visto como la edad del perfil de sus usuarios es cada vez menor y actualmente podríamos decir que Tuenti es una red para adolescentes y niños. Esto provoca que se preste una especial atención a casos de vulneración de la privacidad y de la legalidad en todos los aspectos: desde insultos, publicación de fotos sin autorización, acoso o pornografía infantil.

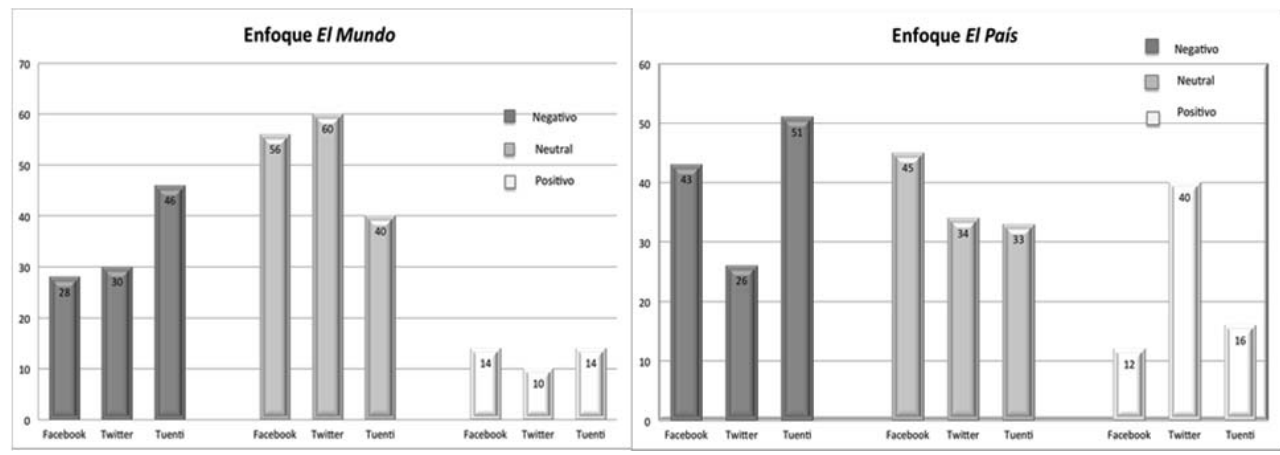

Figura 1. Gráfica comparativa del enfoque de las noticias referentes a cada red social en los dos periódicos analizados. Fuente: elaboración propia. 
A ello se suma además la coincidencia en el tiempo con el caso de Marta del Castillo, que, debido a que los menores implicados y la víctima tenían perfiles en esta red social y sus fotos se usaron en muchos medios de comunicación, elevan esta cifra en el cómputo general, inclinando la balanza hacia una visión desfavorable de Tuenti.

También, aunque justo por lo contrario, resulta muy llamativo el caso del reflejo de Twitter, sobre todo en El País, con un $40 \%$ de noticias positivas. Twitter se ha convertido en la red social de moda, en la que supuestamente está todo el mundo que tiene algo que decir. Quizá por esa vinculación a las últimas tendencias, la visión que se transmite de esta red social es mejor. Además, al estar basada en el microbbloging, no propicia una exhibición de la propia identidad tan completa como Facebook, por lo que apenas presenta problemas con la privacidad, lo que también hace mejorar la imagen que de ella se nos presenta.

\subsection{Temáticas}

La categoría más recurrente en ambos periódicos es Empresarial, lo que podemos atribuir a que una gran cantidad de noticias provienen de agencias y responden sin más a la cobertura de movimientos empresariales de las redes sociales, desde despido o contratación de directivos, a presentación de nuevos proyectos y estrategias, estimación de su capital, beneficios etc.

Los siguientes epígrafes mayoritariamente representados son los relacionados con Tecnología y aplicaciones, muy seguido del de Usuarios. Resulta lógico que las novedades tecnológicas estén a la orden del día en las redes sociales, y por su parte, la audiencia llega muchas veces a los medios convencionales debido a que protagonizan hechos curiosos en las redes sociales.

Como puede observarse en el gráfico, en los dos periódicos el porcentaje de noticias correspondiente a cada categoría planteada discurre paralelamente. Sin embargo, se observa que en el caso de El Mundo las informaciones relacionadas con el ítem Privacidad resultan levemente más frecuentes, con un $14 \%$, en comparación con el caso de El País. En todo caso, tanto en un diario como en otro, la mayor parte de las noticias relacionadas con este ítem guardan relación con los continuos cambios en las condiciones de privacidad de las redes -sobre todo por parte de Facebook- de modo que las informaciones reflejan a usuarios especialmente sensibilizados por este aspecto (sobre todo con la utilización de la información de perfil registrada con fines comerciales).

Abundando más en esta cuestión, si nos centramos específicamente en cada una de las tres redes sociales, podemos observar cómo, por ejemplo, en El Mundo sería Tuenti, con el $22 \%$ de noticias sobre esta materia, la red social más vinculada a problemas de Privacidad. Sin embargo, para El País, Facebook es la red más asociada a informaciones relativas a la privacidad, alcanzando una cifra del 19\% y además vista casi siempre desde un enfoque muy crítico que hace aumentar la visión negativa de Facebook ofrecida por El País, frente a sólo un 2\% tanto en Twitter como en Tuenti. Pero a su vez Facebook también es la red social con un porcentaje más alto de noticias sobre Tecnología y aplicaciones, debido a la enorme cantidad de novedades que genera en su red social prácticamente a diario. 


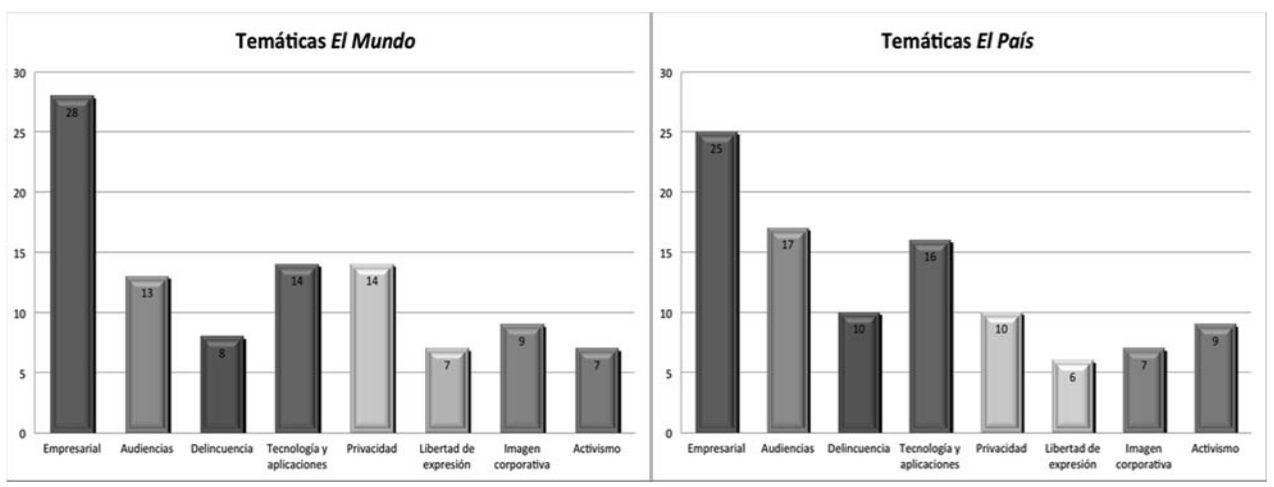

Figura 2. Clasificación temática de las noticias sobre redes sociales en los dos diarios analizados. Fuente: Elaboración propia.

El caso de Twitter en El País también resulta significativo. Como hemos comentado, Twitter tiene una personalidad como red social muy marcada, y sobre todo muchas celebridades están haciendo uso de ella en muchos aspectos. Esto provoca que el cómputo de Usuarios - que engloba, entre otras, las noticias sobre estas personalidades- ascienda hasta el $26 \%$ en el caso de El País, convirtiéndose en el tema más recurrente dentro de este periódico cuando se trata de la red de microblogging. Algo similar ocurre en el caso de El Mundo donde al hablar de Twitter, la temática Usuarios se sitúa con un número de noticias muy alto, un $20 \%$, pero por debajo de Empresarial, con un $29 \%$.

En Tuenti, como ya hemos comentado, sus características y el tipo de noticias a las que se asocia, provocan que el tema de Delincuencia sea el más frecuentemente representado para esta red en El País, con un 25\%. En el caso de El Mundo las piezas que asocian Tuenti a delitos resultan algo más bajas, pero también significativas, con un $19 \%$.

\subsection{Secciones}

En este apartado no podemos comparar equilibradamente los dos periódicos pues algunas secciones varían de un periódico a otro. Pero, a grandes rasgos, podemos afirmar que son más o menos similares. Más de la mitad de las noticias se ubican dentro de las secciones específicas de tecnología dentro de estos periódicos, así El País ubica el $67 \%$ de las noticias en su sección Ciberpaís y en El Mundo el $68 \%$ se sitúan en su sección Navegantes y Tecnología.

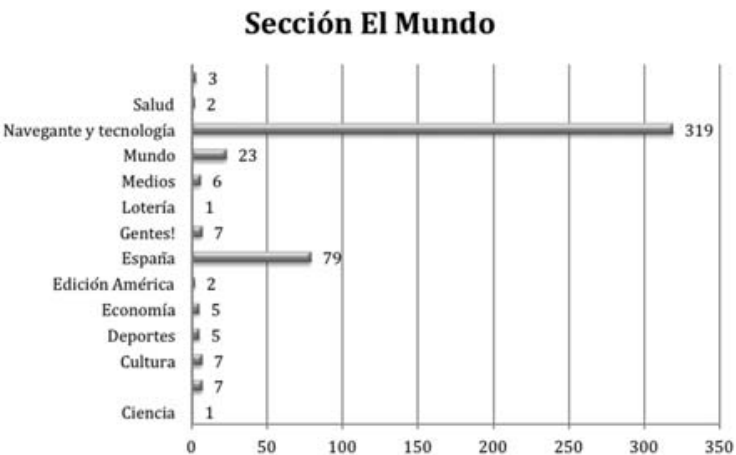

Figura 3. Ubicación de las noticias sobre redes sociales analizadas en El Mundo. Fuente: Elaboración propia. 
Las siguientes secciones con más noticias sobre redes sociales siguen a las arriba mencionadas muy de lejos y se trata de las dedicadas a información nacional: El País cuenta con un $6 \%$ y El Mundo un $17 \%$ en su sección España.

Para poder explicar esta llamativa diferencia en las secciones tenemos que fijarnos en el cómputo general de cada red social. Así observamos como en El Mundo, Tuenti ocupa el $61 \%$ de las noticias dentro de la sección España frente al 34\% de la sección de noticias tecnológicas, que es donde este tipo de noticias se ubica habitualmente. El resto de las redes sociales en este periódico obtiene unos datos muy parecidos a los generales.

Este dato tan alto en la sección nacional del periódico El Mundo con la red social Tuenti se explica nuevamente por el tipo de noticias que acapara. Como hemos explicado esta red social está siempre muy relacionada con temas ligados a los sucesos, situados mayoritariamente en las páginas de información nacional, lo que aumenta

\section{Sección El País}

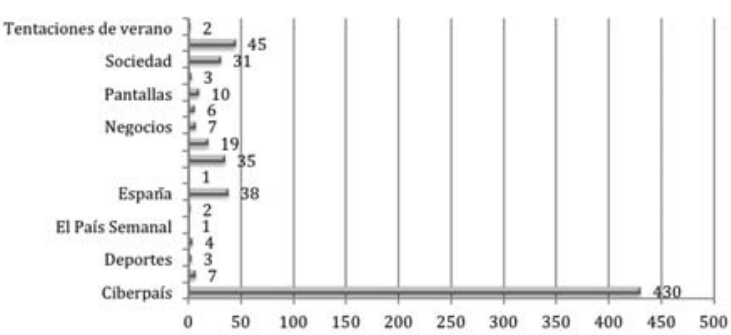

Figura 4. Ubicación de las noticias sobre redes sociales analizadas en El País. Fuente: Elaboración propia. notablemente este dato.

En El País podemos observar esta tendencia pero de forma mucho más atenuada. Así, Tuenti obtiene un 53\% de noticias ubicadas en la sección Ciberpaís frente al 22\% en España. Aunque la sección Sociedad también tiene un dato muy alto, un $15 \%$, fruto también del tipo de noticias que aparecen sobre Tuenti.

\section{Conclusiones}

La cantidad de noticias publicada sobre redes sociales experimenta un aumento espectacular, desde las primeras alusiones del año 2006, donde la mención de redes sociales es meramente anecdótica. A partir de los años 2007 y 2008 comienza a detectarse una presencia más o menos estable, y ya en 2009 y 2010 las noticias sobre redes sociales se disparan, alcanzando unas cifras en torno al centenar de piezas anuales. Gran parte se debe a la atención que concitan las informaciones generadas en torno a Facebook, aunque resulta significativa la progresión de Twitter, que a pesar de llegar más tarde, se ha asentado de tal manera que, en apenas dos años, resulta objeto de interés mediático, en paralelo a la aceptación conseguida entre los internautas.

La postura que adoptan los periódicos seleccionados resulta muy reveladora ya que en casi la mitad de las informaciones analizadas predomina una visión negativa de las redes sociales, asociándolas a una perspectiva más enfocada a lo sensacionalista. A ello contribuye la especial inclinación por dibujar a Tuenti como una red social "peligrosa", de la que hay que proteger a los menores, asimilándola a usos perniciosos de este nuevo instrumento de comunicación. Por el contrario, Twitter cosecha una visión más positiva, más ligada a lo profesional, a la modernidad y a las tendencias. En un plano intermedio, se puede decir de Facebook que, en resumidas cuentas, constituiría la red social por excelencia. 
Es llamativo que dentro de las categorías temáticas en que se pueden clasificar las diferentes noticias abordadas predominen las que aluden a los movimientos empresariales de las distintas redes. No deja de resultar paradójico que, para la prensa, la protagonista sea la red social como institución por encima de los usuarios, centro del modelo de comunicación participativo que encarnan.

En rasgos generales, la manera de abordar estas noticias adolece de cierta falta de profundidad, muchas de ellas son incluso difíciles de verificar y aparecen descontextualizadas, debido a su procedencia dispersa y a lo reciente del fenómeno. La pujanza del mismo parece obligar a incluir noticias relacionadas con este ámbito sin tener en cuenta que cumplan determinados criterios de calidad.

\section{Referencias bibliográficas}

ALEXA (2012): “Top sities in Spain”. http://www.alexa.com/topsites/countries/ES. [Fecha de consulta: 31 de octubre de 2011]

ALEXA (2012): “Top sities". http://www.alexa.com/topsites. [Fecha de consulta: 24 de octubre de 2011]

ASOCIACIÓN DE INVESTIGACIÓN DE LOS MEDIOS DE COMUNICACIÓN (2001): XIII Navegantes en la red. Febrero 2011: http://www.aimc.es. [Fecha de consulta: 31 de octubre de 2011]

BACALLAO PINO, LÁZARO M. (2010): "Representaciones mediáticas de las redes sociales: un estudio de casos", en Revista Latina de Comunicación Social, 65, páginas 114 a 125. La Laguna (Tenerife): Universidad de La Laguna. http://www.revistalatinacs.org/10/art/887_UZaragoza/09_Lazaro_Bacallao.html. [Fecha de consulta: 31 de octubre de 2011$]$

BEAUMONT, Claudine (2009, 16 de enero): "New York plane crash: Twitter braks the news, again". En The Telegraph: http://www.telegraph.co.uk/technology/twitter/4269765/New-York-plane-crash-Twitter-breaks-the-news-again.html. [Fecha de consulta: 31 de octubre de 2011]

CACHIA, Romina (2008): "Los sitios de creación de redes: aspectos sociales", en Telos: Cuadernos de comunicación e innovación, $\mathrm{N}^{\mathrm{0}} 76$, pp.69-84.

CAMPOS FREIRE, Francisco (2008): "Las redes sociales trastocan los modelos de los medios de comunicación tradicionales", en Revista Latina de Comunicación Social, 63, páginas 287 a 293. La Laguna (Tenerife): Universidad de La Laguna. http://www.ull.es/publicaciones/latina/_2008/23_34_Santiago/Francisco_Campos.html. [Fecha de consulta: 31 de octubre de 2011]

COMSCORE (2010): "El tiempo en la Red crece en España un 17\% en mayo, influido por los acontecimientos nacionales e internacionales" http://www.comscore.com/esl/Press_Events/Press_Releases/2011/7/comScore_Releases_Overview _of_European_Internet_Usage_for_May_2011.[Fecha de consulta: 24 de octubre de 2011] 
COMSCORE (2010): "Facebook Captures Top Spot among Social Networkking Sites In India". http://www.comscore.com/Press_Events/Press_Releases/2010/8/Facebook_Captures_Top_Spot_among_Social_Networking_Sites_in_India [Fecha de consulta: 24 de octubre de 2011]

GARCÍA ORTA, María José y MANCINAS CHÁVEZ, Rosalía (2010): "Hacia una nueva comunicación digital y participativa. Blogosfera y redes sociales en los periódicos on line”. En II Congreso Internacional AE-IC Málaga 2010. Comunicación y desarrollo en la era digital. http://www.aeic2010malaga.org/upload/ok /439.pdf. [Fecha de consulta: 31 de octubre de 2011]

RAMONET, Ignacio (2004): "El quinto poder: Información, comunicación y globalización", en Chasqui. Revista Latinoamericana de Comunicación, $\mathrm{n}^{\circ} 88$. http://chasqui.comunica.org/content/view/75/57/. [Fecha de consulta: 24 de octubre de 2011]

SÁNCHEZ CARBALLIDO, Juan Ramón (2008): “Perspectivas de la información en Internet: ciberdemocracia, redes sociales y web semántica". Zer 13-25 (2008), pp. 61-81.

THE COCKTAIL ANALYSIS (2011): "Informe de resultados. Observatorio Redes Sociales $3^{a}$ Oleada". http://www.tcanalysis.com/uploads/2011/02/ObservatorioRedesSociales2011.pdf. [Fecha de consulta: 24 de octubre de 2011] 